\title{
LEARNING FROM IMAGES
}

\section{Perinephric Lymphoma}

\author{
Pramod Gupta $^{*}, 1$, Tushar J. Vachharajani ${ }^{2}$ and Manoj Ketkar ${ }^{1}$
}

${ }^{I}$ Radiology Department, Dallas Veterans Affairs Medical Center, Dallas, TX, USA

${ }^{2}$ Nephrology Section, W. G. (Bill) Hefner Veterans Affairs Medical Center, Salisbury, NC, USA

Keywords: Lymphoma, perirenal lymphoma, perinephric disease, tumor, renal imaging.

Renal imaging plays a valuable role in determining the etiology of renal failure due to intrinsic or extrinsic obstruction of the urinary tract. We report an interesting finding encountered on routine renal imaging in an asymptomatic elderly man who presented with renal failure of unknown etiology. Routine renal ultrasound imaging revealed a large mass around the kidney, which on magnetic resonance imaging of the abdomen was characterized as a retroperitoneal mass that was soft and pliable that surrounded but did not compress or obstruct the retroperitoneal vessels (Fig. 1). However, it caused partial

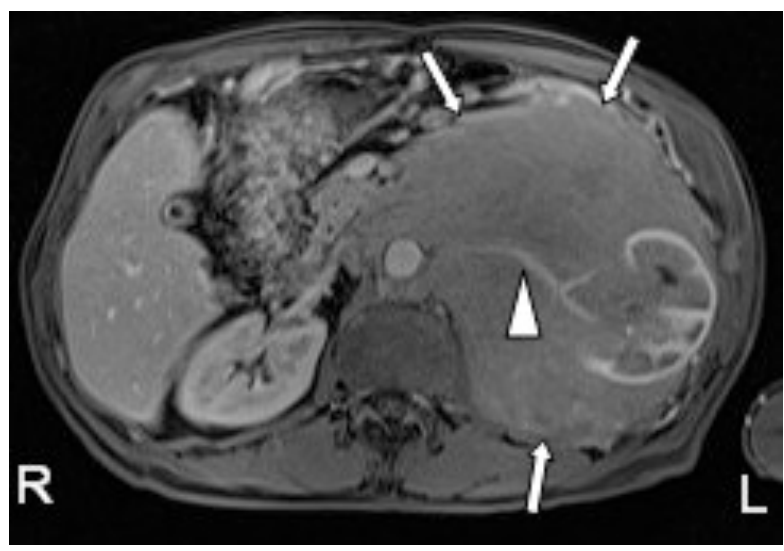

Fig. (1). A 79-year-old male patient was incidentally found to have a large retroperitoneal mass on renal ultrasonography. Contrast enhanced magnetic resonance $\mathrm{T} 1$ axial image with fat suppression, showed a large hypovascular left retroperitoneal mass (arrows) predominantly involving the perinephric space and displacing the left kidney. The mass encases the left renal artery (arrowhead) and aorta, without causing any occlusion. These features strongly favor the diagnosis of lymphoma over renal cell carcinoma, which is usually hypervascular that displaces or invades the vasculature. A biopsy of the mass revealed it to be marginal zone lymphoma. The left kidney shows slightly decreased perfusion and mild hydronephrosis as compared to the normal right kidney.

*Address correspondence to this author at the Radiology Department, Dallas VA Medical Center, 4500 South Lancaster Road, Dallas, TX -75216, USA; Tel: 214-857-0185; E-mail: Pramod.Gupta@va.gov compression of the ureter and renal pelvis resulting in hydronephrosis (Fig. 2). The primary consideration was renal lymphoma. The differential diagnosis of a perirenal mass includes malignant conditions such as renal cell carcinoma, sarcoma arising from renal capsule, metastastic lesion, and benign conditions such as perinephric hematoma, retroperitoneal fibrosis, amyloidosis, and extramedullary hematopoiesis.

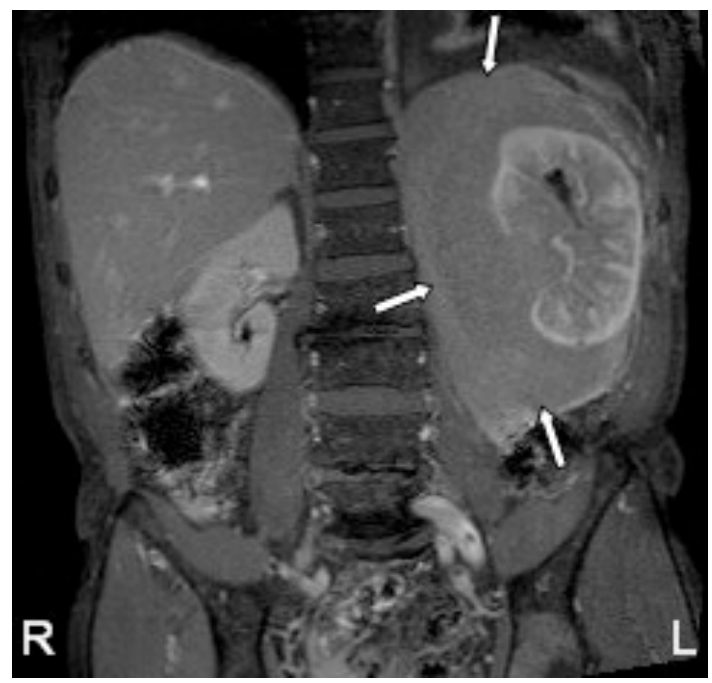

Fig. (2). Contrast enhanced magnetic resonance T1 coronal image with fat suppression, showing the extent of the hypovascular left perinephric mass (arrows).

Primary renal lymphoma is rare, and is usually seen in the setting of a widespread disease. The lymphomatous involvement of the genitourinary organs is second only to hematopoietic and reticuloendothelial tissues. Autopsy diagnosis of renal lymphoma is common compared to finding it on renal imaging (15-50\% vs 3-8\%) [1].

Various findings seen on renal imaging due to renal lymphoma include [2] -

- Multiple parenchymal hypovascular masses of variable sizes in $50-60 \%$ of cases

- Contiguous extension to the kidney or perinephric space from a large retroperitoneal mass in $25-30 \%$ of 
cases. These patients are generally immunocompromised and have widespread lymphomatous bulky tumors.

- As a solitary hypovascular mass in $10-25 \%$ of cases.

- Rarely, diffuse infiltration of the renal interstitium by malignant lymphocytes leading to global renal enlargement without distortion of the normal renal shape on imaging studies.

- Isolated perinephric lymphoma is unusual $(<10 \%$ of cases) and is seen as a rind of homogenous hypovascular soft tissue surrounding the kidney.

- Cystic lesions and tumors predominantly affecting the renal sinus and collecting system are very rare.

Unless the renal lesion manifests in the setting of a widespread lymphoma, percutaneous biopsy with ultrasound guidance is indicated to differentiate lymphoma from other etiologies such as metastatic disease, hypovascular renal cell carcinoma, uroepithelial carcinoma, or atypical infection. Additional immunohistochemical techniques allow accurate diagnosis and characterization of renal lymphoma.

\section{CONFLICT OF INTEREST}

The authors confirm that this article content has no conflict of interest.

\section{ACKNOWLEDGEMENTS}

Declared none.

\section{REFERENCES}

[1] Westphalen A, Yeh B, Qayyum A, et al. Differential diagnosis of perinephric masses on CT and MRI. AJR Am J Roentgenol 2004; 183: $1697-702$.

[2] Sheth S, Ali Syed, Fishman E. Imaging of renal lymphoma: Patterns of disease with pathologic correlation. Radiographics 2006; 26: 1151-68 